\title{
Infancias trans: otra disrupción en las escuelas de la modernidad
}

Trans Childhood: Another Disruption in Modernity Schools

Infâncias trans: outra ruptura nas escolas modernas

Veronica Stewart* iD orcid.org/0000-0002-6201-2336
Mariano Narodowski** iD orcid.org/0000-0002-3122-052X
Maria Delfina Campetella*** iD orcid.org/0000-0001-5412-3717

Para citar este artículo: Stewart, V., Narodowski, M. y Campatella, M. (2O21). Infancias trans: otra disrupción en las escuelas de la modernidad. Revista Colombiana de Educación, 1/82), 263-280. https:/doi.org/ 10.17227/rce.num82-10804

(c) (5) $\begin{array}{r}\text { Recibido: } 28 / 11 / 2019 \\ \text { Ev } \\ \text { Evaluado: } 26 / 05 / 2020 \\ 05 / 05 / 2020\end{array}$

* Licenciada en Periodismo, Universidad de Ciencias Empresariales y Sociales. Asistente de investigación, Universidad Torcuato Di Tella, Argentina. Correo: veronicamstewart@gmail.com

** Doctor en Educación, Universidade Estadual de Campinas. Profesor Titular Universidad Torcuato Di Tella, Argentina. Correo: mnarodowski@utdt.edu

*** Licenciada en Ciencia Política y Gobierno, Universidad Torcuato Di Tella. Asistente de investigación, Universidad Torcuato Di Tella, Argentina. Correo: mdcampetella@gmail.com 


\section{Resumen}

Este artículo de reflexión pretende exponer las consecuencias, en términos teóricos, de la presencia de alumnos trans en las escuelas. El supuesto es que la nueva situación es el resultado de nuevas posiciones de niños y adultos y de una consecuente transformación en la relación de autoridad y poder que media entre ambos, y se busca particularmente comprender el virtual impacto en la tecnología escolar de los altos niveles de autonomía infantil decurrentes de una decisión tan radical como la de modificar la identidad de género. Se parte del concepto de infancia de Ariés (1960), de tecnología escolar de Narodowski (1994) y de performatividad del género de Butler. Se argumenta que la escuela conforma y es conformada por el concepto moderno de infancia a la vez que tiene un rol protagónico en la construcción de género de sus estudiantes. El estudio concluye que el alumnado trans se presenta como disruptivo para la tecnología escolar dado que su sola presencia atenta contra el concepto de infancia heterónoma y vulnerable y contra el concepto biologicista e inmutable de género que la escolarización había integrado a su devenir histórico.

\section{Palabras clave}

Derechos de los niños educación; escuelas; personas LGBTQ; relación estudiante-escuela

\section{Keywords}

Children's rights; education; LGBTQ people; schools; student school relationship

\begin{abstract}
This article attempts to illustrate the theoretical consequences of the presence of trans students in schools. It's assumed that this new situation arises from the new roles played by adults and children, and from the resulting transformation in the authority and power relation that mediates between them. It seeks to comprehend the virtual impact this situation has in school technology insofar making such a radical decision as changing their gender identity gives children high levels of autonomy. This article argues that school both makes up and is made up by childhood while it also plays a key role in their students' gender subjectivity. The study concludes that trans students are disruptive for school technology since their mere presence contradicts the school notion of childhood as heteronomous and vulnerable, and gender as biologically determined and thus, unalterable.
\end{abstract}

\section{Resumo}

Este artigo de reflexão tem como objetivo expor as consequências, em termos teóricos, da presença de alunos trans nas escolas. O pressuposto é que a nova situação é o resultado de novas posições de crianças e adultos e de uma consequente transformação na relação de autoridade e poder que os medeia, sendo particularmente procurado compreender o impacto virtual na tecnologia escolar de alto nível. de autonomia infantil resultante de uma decisão tão radical como a de modificar a identidade de gênero. Parte-se do conceito de infância de Ariés (1960), da tecnologia escolar de Narodowski (1994) e da performatividade de gênero de Butler. Argumenta-se que a escola se conforma e é moldada pela concepção moderna de infância ao mesmo tempo em que ocupa um lugar de destaque na construção do gênero de seus alunos. $O$ estudo conclui que os alunos trans são apresentados como perturbadores da tecnologia escolar, pois sua mera presença ameaça o conceito de infância heterônoma e vulnerável e contra o conceito biologicista e imutável de gênero que a escolarização integrou à sua evolução histórica.

\section{Palavras-chave}

Direitos da criança; educação; escolas; pessoas LGBTQ; relação aluno-escola 


\section{Introducción}

En el 2012 se sancionó en la Argentina la Ley 26.743 de identidad de género, una normativa pionera respecto a la igualdad de derechos en el mundo. La norma establece que toda persona tiene derecho al reconocimiento de su identidad de género, al libre desarrollo de su persona y a ser tratada de acuerdo con esta. En el ejercicio de estos derechos, la ley habilita a toda persona a actualizar su documento de identidad de acuerdo con el género autopercibido, y habilita el acceso a intervenciones quirúrgicas y tratamientos hormonales para quienes deseen adecuar su cuerpo al mismo.

Para el caso de quienes no alcanzaron la mayoría de edad, que en la Argentina es de 18 años, la ley contempla que los menores puedan efectuar el cambio en el registro y el acceso a tratamientos médicos a través de sus representantes legales y con expresa conformidad del menor. También se establece que deberá respetarse la identidad del género adoptado por niños, niñas y adolescentes que utilicen un nombre de pila distinto al consignado originalmente en su documento de identidad. Más aún, determina que, a solo requerimiento del menor de edad, el nombre de pila elegido deberá ser utilizado para la citación, el registro, el legajo, el Ilamado y cualquier otra gestión y servicio, tanto en el ámbito público como en el privado.

Este es un fenómeno muy profundo y con consecuencias sobre todo en la dilución de las identidades fijas sobre las que reposaban la organización y el ethos de diversas instituciones de la modernidad. Nuestro objetivo es partir de la coyuntura de la sanción de la ley para analizar las cuestiones históricas que la sostienen y la retroalimentan, especialmente en el espacio escolar.

En las escuelas, el nuevo escenario legal abre la posibilidad de que entre los alumnos se encuentren niños y niñas que tomen una decisión activa sobre su identidad de género autopercibida, y que, sin ningún otro requerimiento, los docentes, los directivos, los otros alumnos y el conjunto de la comunidad escolar se vean legalmente obligados a reconocer y a respetar la decisión.

Este cambio legal parece el epifenómeno de tendencias políticas preexistentes. En Argentina, el movimiento LGBT+ ya había obtenido una ley de matrimonio igualitario en el 2010 (Ley 26.618). La ley de identidad de género consagra más derechos a las personas trans (incluidos los niños y las niñas). ${ }^{1}$ Pero no se trata solo de una cuestión jurídica. Según Saldivia

1 La Ley 26.618, también conocida como ley de matrimonio igualitario, fue sancionada en el año 2010 y extendió el derecho al matrimonio civil a personas del mismo sexo, convirtiendo a Argentina en vanguardia respecto al resto de América Latina y Estados Unidos. La Ley 26.743, sancionada en el 2012, cobró especial relevancia por ser aprobada por unanimidad en el Senado y por constituirse como la primera ley de identidad de género en el mundo que no patologiza las identidades trans. Aunque la sanción de ambas leyes generó fuertes debates no exentos de conflicto, en particular entre activistas del movimiento LGBT+ y la Iglesia católica, la experiencia argentina ha tomado especial relevancia por sus destacados avances normativos en materia de derechos LGBT+ (Tabbush et ál., 2016) 
Menajovsky (2016), al incluir en sus postulados el derecho a la identidad de género de los menores de edad, la ley acoge recientes cambios paradigmáticos en las ciencias jurídicas respecto de su entendimiento sobre niños y niñas. Siguiendo a la autora, en el ámbito jurídico la infancia estuvo históricamente gobernada por el complejo tutelar, modelo según el cual:

la niñez no era considerada sujeto de derecho, sino que, por el contrario, se la definía por sus carencias y era objeto de protección y control por parte del Estado, la familia y la sociedad, quienes debían brindarle tutela y asistencia. (2016, p. 81)

\section{Las infancias trans en la escuela como problema teórico}

En la literatura académica internacional, la investigación trata de comprender el fenómeno en términos del par discriminación-no discriminación (Payne y Smith, 2014), argumentando a favor de escuelas más inclusivas y aun más cómodas para los alumnos trans (Burt et ál., 2010) e identificando los mejores mecanismos de formación y capacitación docente (Kintner-Duffy et ál., 2012) o el más sano vínculo con los progenitores (Rahilly, 2015). Si bien estos estudios son muy necesarios, el presente trabajo pretende dar un paso adelante, en una dirección teórica que busca entender claves no siempre visibles en el seno de las instituciones escolares.

El supuesto de este artículo es que la nueva situación es el resultado de nuevas posiciones de niños y adultos y de una consecuente transformación en la relación de autoridad y poder que media entre ambos (Mead, 2002; Postman, 1994). Por consiguiente, pretende exponer las consecuencias, en términos teóricos, de un escenario escolar en transformación. Particularmente, interesa comprender el virtual impacto en la tecnología escolar de los altos niveles de autonomía infantil derivados de una decisión tan radical como la de modificar la identidad de género.

La hipótesis de trabajo es que la infancia trans disrumpe la tecnología escolar poniendo en jaque los principales dispositivos sobre los que asienta su funcionamiento: el concepto mismo de infancia y el de ser alumno y la posición del docente como lugar del saber. A su vez, también evidencia el rol de la escuela en la construcción de género de sus estudiantes.

El concepto de disrupción, pues, atraviesa los argumentos del presente estudio. Como señala Campanella (2018) la última y más importante característica de la disrupción es estar desajustada: no es funcional porque crea una distancia, una diferencia, respecto de la lógica anterior que sostenía a las partes combinadas en una estructura. Pero esa fisura se parece más a una fuga: una sustitución o nominación (una mutación) que hace pedazos la estructura y la altera, la convierte en otra. 
¿Son las infancias trans un fenómeno que anticipa o predice cambios radicales en las instituciones escolares?

\section{Escuela e infancia: el dispositivo de infancia}

A partir de estudios previos, podemos afirmar que nuestros sentimientos modernos de infancia constituyen una construcción histórica propia de la modernidad. Tras analizar los aportes producidos por la historia de la educación y las evidencias brindadas por la demografía histórica francesa, en especial aquellas presentes en la obra de Philippe Ariés, L'Enfant et la vie familiale sous I'ancien regime (1960), diferentes autores señalan que hasta la Edad Media nuestros actuales sentimientos de infancia no existían en la cultura occidental: los niños no inspiraban ternura ni instinto de cuidado, compartían todas las actividades a la par de los adultos, y no se diferenciaban mayormente de ellos ni por sus vestimentas, sus juegos, sus tareas ni por los trabajos que realizaban.

Siguiendo a Baquero y Narodowski (1994), esta situación cambió hacia el inicio de la modernidad europea, cuando el niño comenzó a ser visto como un sujeto con características específicas, en particular un sujeto carente de los conocimientos necesarios para desenvolverse por sí solo en el mundo. De esto se desprende que se les deba amor y cuidado: el Ilamado sentimiento bifronte de infancia (Ariés, 1986).

Este cambio en la conceptualización de la infancia es la base de la escuela moderna. Como señala Narodowski, "la pedagogía obtiene en la niñez su excusa irrefutable de intervención para educar y reeducar en la escuela, para participar de la formación de los seres humanos y de los grupos sociales" (1994, p. 26). Los niños (los alumnos), por dependientes y heterónomos, deben asistir a la escuela. Allí los espera un adulto (el docente) a quien deberán obedecer, ya que es quien, desde su lugar del saber, los guiará a una situación de autonomía.

Por otro lado, podría conjeturarse que esta escuela moderna está incluida en lo que Mead (2002) identifica como una cultura posfigurativa, en la cual los niños aprenden primordialmente de sus mayores. Allí los cambios son tan lentos e imperceptibles que el pasado, es decir, la experiencia de los mayores y la tradición, proporcionan las pautas básicas para la vida futura de los niños. Extrapolado a la escuela, esto justifica la obediencia por parte de los alumnos hacia sus docentes: los segundos poseen el conocimiento que los primeros carecen y necesitan para poder vivir (Narodowski, 2016).

En suma, la infancia es un concepto histórico definido por características vinculadas a la carencia, como la ignorancia (carencia de saber) y la heteronomía (carencia de moral propia). La pedagogía moderna, concebida 
en una cultura posfigurativa, naturaliza este concepto de infancia y lo reinserta en un ámbito particular, la escuela, para construir tanto en forma teórica como práctica, el concepto de alumno y, en contraposición, el concepto de docente. En otras palabras, ser alumno en la institución escolar moderna es básicamente ocupar un lugar heterónomo de no-saber, contrapuesto a la figura del docente, un adulto autónomo que sabe (Narodowski, 1999). Cuando hablamos de infancia y escuela hablamos del dispositivo de infancia, pieza fundamental de la tecnología escolar.

\section{Escuela y género: la escuela como constructora de género}

Así como la infancia es una noción construida a lo largo de la historia, las autoras clásicas en la teoría feminista — desde Simone de Beauvoir (1981 [1949]), hasta Judith Butler (1990) — afirman que el género es también un constructo sociocultural. Según Lamas (2000), el género fue conceptualizado por esta corriente como

el conjunto de ideas, representaciones, prácticas y prescripciones sociales que una cultura desarrolla desde la diferencia anatómica entre mujeres y hombres, para simbolizar y construir lo que es "propio" de los hombres (lo masculino) y "propio" de las mujeres (lo femenino). (2000, p. 2)

Para esta vertiente feminista, a partir de esta clasificación cultural se definen no solo la división del trabajo, las prácticas rituales y el ejercicio del poder, sino también las características que se consideran exclusivas a uno y otro sexo en materia de moral, psicología y afectividad. En palabras de Tomasini: "el género opera [...] como un significante binario de distribución social jerárquica de potencialidades, expectativas, atributos, históricamente desarrollados por los seres humanos" (2008, p. 97).

Dentro de la corriente feminista que entiende al género como un constructo social, Butler (1990) sostiene la teoría de la performatividad. La misma sostiene que, además de las influencias sociales que construyen el concepto de género, los sujetos también lo reconstruyen: el género es "el resultado de un proceso mediante el cual las personas recibimos significados culturales, pero también los innovamos" (2001, p. 18). Sabsay (2009) resume los puntos clave del pensamiento de Butler: el género es un efecto, una actuación, un hacer, y no un atributo con el que cuentan los sujetos. Se trata de una práctica social reiterativa. Esta se realiza en función de normas sociales que nos exceden: el sujeto no es dueño de su género, y no realiza simplemente la performance que más le satisface, sino que lo actúa en función de normativas genéricas que promueven, legitiman, sancionan y excluyen. Sin embargo, dado 
que el género no existe por fuera de esta actuación o performance, y que las normas de género no son sino la propia reiteración y actuación de esas mismas normas, estas están siempre sujetas a la resignificación y a la renegociación (Sabsay, 2009).

Uno de los espacios donde se disputa el poder sobre dichas significaciones y resignificaciones es la escuela. Tal como señala Elizalde, esta se entenderá como "un dispositivo cultural y político estructurado y transversalizado por relaciones de poder que intentan regular y modelar los cuerpos y comportamientos de sus integrantes de acuerdo a sus modos hegemónicos de saber/poder, así como a sus valores y deseos" (2014, p. 46). En términos de género, estos modelos hegemónicos son la heteronorma y la cisnorma, dado que, a pesar de que la mujer haya sido incorporada a los sistemas educativos hace décadas, estos fueron concebidos bajo un orden patriarcal (Beer, 2008). Esto quiere decir que aquellos alumnos que se definan como heterosexuales y que sientan que su identidad de género se condice con su sexo biológico encontrarán en las narrativas impartidas por la escuela suficientes herramientas para identificarse y definirse. A su vez, dichas narrativas acabarán por definirlos y moldearlos, aun en una lógica patriarcal. Sin embargo, todos aquellos que se encuentren por fuera de esa definición significarán una disrupción para todo eso que la escuela busca perpetuar. Así, "la escuela genera un espacio narrativo privilegiado para algunas formas de identidad y un espacio de desigualdad y subordinación para otras" (Elizalde, 2014, p. 45).

En palabras de Hernández y Reybet, la escuela reduce el género a una diferencia sexual y lo contempla, por lo tanto, como un elemento natural y no social. Como bien señalan las autoras, "en tanto no problematizadas, estas relaciones se inscriben en el campo de la diversidad, espacio natural de la diferencia como valor que elude contemplar las relaciones de desigualdad" (2006, p. 4). A esta invisibilización de la diversidad Ilevada a cabo mediante dispositivos escolares que actúan en pos de la heteronorma se la denomina, en términos de Sedgwick (1990), pedagogía del clóset. Algunos de estos dispositivos son el currículo y el control de los cuerpos, observable en el uso de los baños, las clases de educación física y la disposición de los alumnos en el aula y el patio.

Como destaca Elizalde, la escuela ejerce una clara "pedagogía de la sexualidad y disciplinamiento de los cuerpos" (2014, p. 33). En este sentido, la autora afirma que la herramienta más efectiva de la que disponen las instituciones educativas para transmitir ciertas enseñanzas sobre la sexualidad es el currículo oculto que produce normatividades sobre cómo ser hombre y mujer. El mismo es definido por Parga Romero como "el proceso de transmisión de normas implícitas, valores y creencias que subyacen en las formas culturales utilizadas por la escuela" (2008, p. 41). Así, gran parte de lo que los alumnos aprenden sobre su sexualidad en la escuela está mucho más 
ligado a los conocimientos de orden valorativo y normativo que transmite por fuera de sus contenidos oficiales que a una suerte de instructivo explícito dentro del currículo tradicional.

Gran parte de ese currículo no explícito se relaciona con la reproducción de roles de género. Elizalde (2014) afirma que la escuela normaliza estereotipos de género a través de sus actividades, donde separa al alumnado en el par binario mujer/hembra y hombre/macho. Estas categorizaciones ubican a la mujer en roles domésticos y la definen como un sujeto vulnerable y sensible, mientras que el varón se ve asociado al mundo laboral y es presentado como un sujeto fuerte y poderoso (Schargrodsky y Narodowski, 2005). Así, la escuela introduce estas categorías como definiciones inamovibles sobre las que es imposible operar. Como explican Morgade y Alonso: "el cuerpo y el sexo quedan del lado de lo que es o lo que debería ser, mientras que el género se erige dando por sentados esos cimientos, lo cual impide conceptualizar a los cuerpos más allá de la ideología de lo dado" (2008, p. 22).

Sin embargo, los contenidos incluidos en el currículum oficial de todo sistema educativo también operan como dispositivos clave para la construcción de género en la escuela. Tal como señalan Schargrodsky y Narodowski (2005), la masculinidad no es estática ni atemporal; es histórica. No es la manifestación de una esencia interior: se construye socialmente, se crea en la cultura. Así, la escuela enseña a todos aquellos varones cuyas actitudes son leídas como femeninas a deshacerse de esos comportamientos: la institución escolar se encargó de obstruir el desarrollo emocional del varón a fin de que no superase los límites de la normalidad falologocéntrica heterosexual.

Esta categorización y valoración en términos de género, que no solo organiza al alumnado, sino que define sus percepciones y acciones para consigo mismos y para con sus pares, ocurre en la escuela desde el nivel de la educación infantil. Tomasini (2008) define tres niveles para analizar dichos actos de categorización sexual desde la escuela infantil. En primer lugar, los actos institucionales, donde el género aparece como criterio saliente en la organización de las actividades escolares. Entre ellos, la autora destaca la formación en filas, el control de asistencia, la distribución en las mesas de trabajo, el reparto de objetos diferenciados a chicas y chicos (por ejemplo, tarjetas de colores diferentes), etc. En segundo lugar, se encuentran las intervenciones de los distintos agentes institucionales que clasifican y valoran diferencialmente a sus alumnos y alumnas. Por último, la autora se refiere a las acciones del propio alumnado en el ámbito de interacción entre pares, en las cuales se ponen en juego esquemas de clasificación binarios y excluyentes y se operan tipificaciones según criterios de masculinidad/ feminidad. Cabe destacar que este último nivel sucede luego de que los niños y niñas hayan internalizado aquella categorización impuesta en 
los primeros dos niveles. Estas formas constantes de oposición y binarismo no solo imponen valoraciones distintas entre los géneros constantemente, sino que refuerzan la idea de que dicha diferencia entre géneros es esencial $y$, por lo tanto, inmodificable.

Otro de los ámbitos en los que más claramente puede observarse la construcción de género en la escolaridad es en las clases de educación física y en los momentos del recreo. Como señala Beer (2008) en su investigación, los docentes naturalizan la actitud activa del niño como algo propio de su masculinidad. Así, los varones juegan en las zonas centrales del patio de juegos mientras que las niñas realizan sus juegos pasivos en la periferia. Las autoridades escolares no solo lo leen como parte de la naturaleza de los niños y las niñas, sino que no pueden ver cómo la institución escolar misma reproduce esta diferencia. Scharagrodsky (1999) muestra con evidencia etnográfica que en las clases de educación física la distribución de los cuerpos de alumnas y alumnos en el espacio no es equitativa, ya que las primeras ocupan espacios periféricos o marginales, a un costado de donde juegan los varones y en espacios reducidos, en tanto que estos últimos ocupan espacios centrales. En este sentido, Beer explica que la educación física se caracteriza como la aplicación de estímulos pensados para "explotar las capacidades biológicas y alcanzar determinado grado de eficacia deportiva, siempre condicionada por el cuerpo 'dado, por lo que 'es'" (2008, p. 166).

En lo que al disciplinamiento del cuerpo se refiere, la escuela ejerce un firme control sobre su alumnado en el uso de los baños. Tal como las clases de educación física están diseñadas para resaltar la esencia física de cada alumno según su género, Ingrey (2012) argumenta que los baños separados en masculino y femenino aportan de manera similar a la naturalización de dichas categorías de género como fijas y relacionadas directamente al cuerpo biológico. Como destacan Slater et ál., el mensaje es que "existen dos géneros que son opuestos y que se los debe mantener separados cuando desnudos" (2018, p. 954). Por su parte, Ingrey agrega que las puertas y los cubículos remiten a las prácticas foucaultianas de encierro y división, ya que "permiten la entrada de un cuerpo físico a la vez, y así convierten a masas de cuerpos en unidades individuales, manejables y generizadas" (p. 802). Slater et ál. van incluso más lejos, y argumentan que todo lo que se les enseña a los niños con respecto al uso del baño enseña "otras lecciones sobre la identidad y el cuerpo" (2018, p. 953).

Así, estas enseñanzas suceden, también, durante la adultez, aunque de formas mucho menos claras que en los primeros años. Un ejemplo de este disciplinamiento es lo que las autoras Ilaman "vigilancia en el baño" (toilet policing), es decir, todas aquellas prácticas "que reducen la autonomía de los niños y los jóvenes a través de la vigilancia y la intervención a la hora de usar el baño (por ejemplo, no poder usarlo durante horas de clase)" 
(p. 961). Igual que en tantas otras instancias escolares, los cuerpos de los niños son controlados, en este caso, en términos de género y acceso al baño de acuerdo al mismo. Es por eso que cuando un alumno tiene un cuerpo que la escuela no puede clasificar cómodamente dentro del par binario que perpetúa, es decir, "aquellos cuerpos que transgreden límites, cuerpos 'incivilizados' que no usan el baño como queremos que lo hagan" (Slater et ál., 2018, p. 959), la escuela se siente amenazada.

Además de las distintas formas de disciplinamiento con las que cuenta la escuela para regular a su alumnado en términos de género, también debe considerarse el discurso y las acciones de quienes pasan más tiempo con dichos estudiantes: los docentes. Como bien señala Frohard-Dourlent, a pesar de que entendamos la educación como una herramienta de inclusión social, las escuelas constantemente participan de procesos y prácticas institucionales diarias que acaban por excluir a ciertos alumnos; "incluso cuando ejercen cierta resistencia en sus prácticas individuales, los educadores permanecen imbricados en sistemas que refuerzan la conformidad de género y sexo a través de los permisos, las prohibiciones y las expectativas que normalizan en las escuelas" (2016, p. 65).

\section{La disrupción de las infancias trans en la escuela}

Como se vio en el inicio de este estudio, el examen tutelar y restrictivo hacia la infancia por parte de la teoría jurídica ha sido desplazado por un nuevo paradigma que cuestiona la mirada sobre los menores de edad como carentes de capacidad y establece que niños y niñas son titulares y portadores de derechos y atributos que les son inherentes por su condición de persona. Esto se evidencia primeramente en la Convención sobre los Derechos del Niño (Unicef, 1989), la cual prescribe el derecho a formarse un juicio propio y establece el interés del niño como un límite a las decisiones respecto a sí mismo que puedan tomar tanto el Estado como los adultos en general (Saldivia Menajovsky, 2016). La normativa de identidad de género dispuesta en Argentina, entonces, constituye un caso dentro de esta tendencia global.

Pero no se trata solo de cuestiones jurídicas. Al contrario, este cambio de paradigma en las ciencias jurídicas es, en realidad, un reflejo más de esta transformación de la infancia y de la adultez. Procesos sociales y productivos parecen transformar la concepción sobre la infancia tal como fue definida por la modernidad.

Retomando el trabajo de Mead (2002), la autora señala el advenimiento en nuestra sociedad de lo que define como cultura prefigurativa. Esta se caracteriza por la sucesión vertiginosa de cambios tecnológicos y descubrimientos científicos, a diferencia de una cultura posfigurativa, en la que los 
cambios habían de producirse lentos, poco significativos y casi imperceptibles para la vida cotidiana de las personas. Como consecuencia, en una cultura prefigurativa el saber y la experiencia de los adultos se torna inservible ante un futuro impredecible y su legitimidad para imponer su autoridad pierde terreno. El valor ya no reside en la tradición, la antigüedad, la experiencia y el conocimiento sobre el pasado sino en la capacidad de adaptarse a lo nuevo en forma inmediata.

Así como el concepto de adultez se ve alterado, el de infancia también. En una cultura en la que la experiencia previa pasa a ser una carga pesada y lo privilegiado es la capacidad de adaptación, la juventud se convierte en algo deseable y valorado. Esto quiere decir que la infancia ya no se caracteriza por una carencia. Como señala Narodowski (2016) la infancia, la adolescencia y la minoría de edad ya no suponen una discapacidad, sino un punto de referencia que pasa a ser valorado y considerado. Además, los niños parecen percibir que muchas veces sus mayores utilizan medios inapropiados, que su desempeño es penoso y que los resultados son muy inciertos, es decir, ya no encuentran en ellos una fuente certera de conocimiento y de guía. Por lo tanto, su condición de heterónomos pierde sentido y, por el contrario, gana fundamentos su autonomía.

Relacionado con la postura de Mead (2002), Neil Postman (1994) argumenta que fue la llegada de medios masivos de comunicación, en particular de la televisión, lo que ponía en peligro el elemento que, a su entender, define a la infancia: los secretos de los adultos. Según el autor, la distinción entre niños y adultos es que los segundos poseen conocimientos que los primeros no tienen, tanto así que define a los niños como un grupo de personas que desconocen ciertas cosas que los adultos sí saben. Sin embargo, el avance y la difusión generalizada de la televisión habrían de eliminar esa línea divisoria al transmitir conocimientos y pautas culturales en forma equivalente a todos los sujetos, sin importar sus edades. En consecuencia, para Postman se podría afirmar que estamos viviendo la desaparición de la infancia tal como la conocemos.

Esta visión de la infancia posterior a la discapacidad implica una creciente horizontalización del vínculo entre grandes y chicos, lo que a su vez presupone la intercambiabilidad de los roles: la actual cultura prefigurativa sobreentiende una suerte de equivalencia entre las partes que hace que cualquiera pueda ocupar cualquier lugar.

Esto tiene consecuencias directas sobre nuestras expectativas respecto al comportamiento de la infancia: en una cultura posfigurativa, lo lógico era la subordinación de los niños a la autoridad adulta, es decir, la obediencia, ya que ella es la que operativiza en términos socialmente concretos las asimetrías propias del vínculo entre grandes y chicos. Para el caso de las escuelas, la historia de la educación ha mostrado el arduo camino de la legitimidad docente en un entramado de avances y retrocesos en procura 
de una autoridad asimétrica basada en la obediencia. El trabajo de Muñoz Hurtado (2019), por ejemplo, ha brindado evidencia historiográfica al respecto, advirtiendo acerca de la no linealidad del proceso

En una cultura prefigurativa, esta lógica de obediencia se encuentra cuestionada. Por otro lado, es posible identificar el advenimiento de infancias hiperrealizadas: niños que viven en la absoluta inmediatez, con acceso constante a la información y al mundo a través de sus dispositivos digitales. Se trata del efecto de la televisión señalado por Postman (1994), ahora acelerado y multiplicado, con el conocimiento disponible para los niños (Fabris et ál., 2011).

El fenómeno de las infancias trans pone en evidencia estos cambios. Se trata de niños y niñas cuya opinión es considerada legítima y valiosa, con conocimiento acerca de temáticas como identidad y sexualidad; opinión muchas veces más calificada que la que poseen los adultos. Niños que pueden hacer uso de su autonomía y cambiar su nombre de pila de acuerdo a su identidad de género autopercibida, y que tienen derecho a que se les respete en todos los ámbitos, públicos y privados, solo con que expresen que ese es su deseo. Es decir, infantes que pueden tomar la decisión de realizar modificaciones a su identidad y cuerpo y que, por lo tanto, gozan de autonomía sobre ellos.

Por lo tanto, las infancias trans se presentan como una disrupción para la escuela en tanto y en cuanto entran en contradicción con el dispositivo pedagógico de infancia bajo el cual se concibió la tecnología escolar. $\mathrm{Si}$, como ya vimos, nuestra cultura moderna ha sido posfigurativa en los términos de Mead y se ha basado en la construcción de una infancia heterónoma y dependiente de una adultez autónoma y responsable, la presencia de alumnado trans, en cambio, significa la presencia de niños que no cumplen con este supuesto.

Así, las infancias trans desafían el lugar del docente como lugar del saber, en el sentido de que hoy son consideradas como portadores de conocimientos valiosos, por ejemplo, respecto de su propio cuerpo y su propia identidad, tanto así como para poder realizar cambios personales respecto de su género autopercibido: la habilitación de la autopercepción y su puesta en práctica legal es un acontecimiento formidable que muestra el contexto prefigurativo de una hiperrealización infantil.

Ya no es el conocimiento del docente o de los progenitores el punto único de referencia. Posiblemente no lo sean en absoluto. Este desafío al lugar del docente como lugar del saber es mayor aun cuando se trata de infantes que no solo poseen conocimientos considerados valiosos sino que poseen más conocimientos que los mismos docentes. Como señala Beer (2008), las certezas que antes presentaba la escuela son ahora desafiadas por los nuevos movimientos y espacios sociales, que producen otros saberes y conocimientos, como es el caso de la cultura queer y LGTB+. 
La institución educativa pasa de ser el sitio de la norma a uno de perplejidad frente a saberes que disrumpen desde el discurso menos esperado: el del niño de corta edad decidiendo por sí mismo en asuntos centrales para su vida. Al mismo tiempo, este desafío al lugar del docente como lugar del saber implica un desafío a su autoridad. Si la legitimidad de su posición de poder en el aula radicaba antes en sus saberes y conocimientos derivados de su experiencia adulta en contraposición a la carencia característica de los alumnos infantes, ahora esta legitimidad se encuentra al menos cuestionada.

La creciente horizontalidad respecto al vínculo entre niños y adultos y la intercambiabilidad de roles que de esto se deriva tiene su correlato en el fenómeno de la niñez trans escolarizada. Las escuelas, según el mandato legal, deben subordinarse a la voluntad de niños y niñas que deciden realizar cambios respecto a su identidad de género. Son la infancia y la adolescencia ahora las portadoras de una cultura legítima que obliga a sus padres y a sus maestros a adaptarse a ella: ya no es el chico el que debe callar frente a la cultura escolar, sino la escuela la que se adapta a las nuevas situaciones (Narodowski, 2016).

En lo que a las infancias trans se refiere, esto se hace evidente en las decisiones que deben tomar los directivos respecto al uso de los baños — o a la posible reforma edilicia que implicaría la desbinarización de los mismos - el uso de los uniformes y la conformación de los equipos de educación física, entre otros. Como destaca Lenzi en torno a este último punto, "los atletas trans plantean un dilema porque el enfoque de segregación por sexos en la escuela está basado en la afirmación de que los cuerpos masculinos y femeninos son biológicamente diferentes" (2018, p. 853). Por lo tanto, la resolución de esta problemática radica en lo que cada escuela elija priorizar: la supuesta competencia justa, basada en la división de los alumnos respecto a su conformación biológica, o el respeto por la identidad de género de estos.

Sin embargo, incluso aunque la decisión escolar implique no hacer grandes transformaciones de espacios, pero sí permitirles a los alumnos transitar su escolaridad de acuerdo a su identidad de género autopercibida, el mero proceso de decisión pone en evidencia la artificialidad de esta generización llevada adelante a diario, y desde siempre, por la escuela. Se juega tanto la invisibilidad de la diferencia como su hipervisibilidad, lo que se inscribe en la tensión entre el mandato fundacional de igualar con el que se gestó históricamente la escuela, y la exclusión que ejerce sobre diversos contingentes de niños, niñas y jóvenes (Elizalde, 2014).

Así, al ser la escuela un legitimador de identidades jerarquizadas, la presencia de alumnos trans pone en evidencia aquellas identidades por fuera de la norma, es decir, identidades evidentemente construidas y no naturales. Esto es amenazante no solo porque lleva a preguntarse qué 
hacer con ellas, sino porque además expone cómo, si esas identidades disidentes son socialmente conformadas, aquellas que sí responden a la normalidad que la escuela predica también lo son.

En última instancia, y este probablemente sea el principal hallazgo teórico, la mayor amenaza que representan las infancias trans para la escuela no tiene que ver con su tratamiento dentro de la institución educativa, sino con aquello que esta pregona como esencial y natural y este nuevo escenario presenta como construido. De la misma forma en la que dicho alumnado se siente incómodo en la escuela por no hallar en ella un espacio de identificación y construcción de identidad, la misma presencia de alumnos queer, trans o no binarios incomoda, a su vez, al sistema escolar, ya que pone en evidencia sus supuestos más profundos. En otras palabras, la habilitación de un alumno trans en el territorio escolar parece constituir un conflicto no tanto (o no solo) respecto de ese alumno sino de todos los demás en la medida en que una pregunta sobrevuela la escuela: si ese chico o chica pudo construir su genericidad de acuerdo a su propia percepción, ¿por qué no hacerlo todos?

Las infancias trans, por lo tanto, no solo evidencian la construcción social del género llevada a cabo por la escuela, sino que expone a los niños pretendidamente inocentes y heterónomos, como sujetos con una noción clara de su propia sexualidad y las herramientas para tomar decisiones conscientes al respecto. Las infancias trans en las escuelas, en últimas, parecen dificultar severamente las formas tradicionales de censura respecto de la sexualidad y del conjunto de las identidades divergentes, incluso las que no se enraízan en cuestiones de género.

Por lo tanto, se trata de un problema no solo pragmático, sino también teórico: las infancias trans demuestran que aquellas bases sobre las cuales la escuela reproduce la generización de sus alumnos no son naturales, sino más bien sociales y construidas. Esto quiere decir que si la construcción de nuestra subjetividad es un proceso consciente e inconsciente, las instituciones escolares se pueden convertir en un terreno de resubjetivación (Hernández y Reybet, 2006).

\section{Conclusiones}

Las nuevas normativas vigentes en torno a las infancias trans obligan a las instituciones escolares a enfrentarse a la crisis que atraviesan en, al menos, dos de los núcleos que sostienen a la escolaridad moderna desde sus inicios: el de infancia y el de género.

Por un lado, la autonomía que les brinda la ley a los niños trans no se corresponde con la heteronomía supuesta de la infancia; heteronomía necesaria, cabe destacar, para sostener la relación asimétrica entre el 
alumno y el docente y, por lo tanto, la autoridad del segundo sobre el primero. Si la escuela de la modernidad funcionaba con base en combustible adulto, es posible que estas nuevas relaciones — cada vez más entre equivalentes - limiten su capacidad performativa. Como vimos, el lugar del docente como lugar del adulto que sabe fue construido históricamente: disrupciones como las que analiza el presente estudio ponen en perspectiva y prospectiva esta cuestión, especialmente en los desafíos referentes a sostener la autoridad docente en escenarios prefigurativos.

Por el otro, la capacidad de operar sobre el género significa entenderlo como un campo de identificación y resignificación construido y, por ende, modificable. Esto se contradice, a su vez, con el supuesto pregonado tradicionalmente por la escuela y por la pedagogía de que el género se corresponde con la biología y es, en consecuencia, una característica fija e inamovible de cada sujeto. Las infancias trans desnudan estos conceptos como las construcciones sociales e históricas que son y, por lo tanto, abren el campo de juego a nuevos entendimientos sobre ellos.

Se trata del desajuste de la disrupción expresado en un acontecimiento imprevisible; el desajuste de la incorporación de infancias trans a las escuelas no es funcional y crea una distancia prefigurativa; una diferencia respecto de la anterior lógica posfigurativa que sostenía a las partes combinadas en la tecnología escolar. Una fuga (una mutación) que puede hacer pedazos la estructura y alterarla, convertirla en otra.

El estudio de Campanella (2018) trae a Gilles Deleuze y Félix Guattari, quienes desarrollan la idea de línea de fuga: hay ruptura cada vez que de las líneas segmentarias surge bruscamente una línea de fuga, que también forma parte generando la imposibilidad de alternativas sin que la multiplicidad preexistente se transforme según esa línea (Campanella, 2018). La línea de fuga, entonces, imposibilita cualquier reconstrucción de la lógica anterior y desencadena una complejidad dentro de la complejidad, haciendo reproducir conexiones de modo diverso. Ya nada es lo que era.

Las infancias trans, por lo tanto, disrumpen en lo escolar tanto en las líneas de autoridad que suponían una infancia obediente y heterónoma como en las líneas de la homogeneidad generizada, que suponían cuerpos normalizados e irreflexivos. Hemos aportado argumentos en el sentido de que no se trata - únicamente- de cambio inclusivo o de reconocimiento de derechos: en teoría, bien parece que estamos en presencia de otro elemento de fuga que podría suspender las relaciones sociales y de poder de la educación escolar tal y como las conocíamos. Futuras investigaciones empíricas podrán enriquecer y matizar estos hallazgos de la teoría. 


\section{Referencias}

Ariés, P. (1960). L'Enfant et la vie familiale sous l' ancien regime. Plon.

Ariés, P. (1986). La infancia. Revista de Educación, 281, 5-17. https:// es.scribd.com/doc/187776563/Aries-P-1986-La-infancia-en-Revista-de-Educacion

Baquero, R. y Narodowski, M. (1994). ¿Existe la infancia? Revista del Instituto de Investigaciones en Ciencias de la Educación, 6, 2-10.

Beer, D. (2008). Visiones y discursos en la educación física de la escuela primaria. En G. Morgade, y G. Alonso (comp.). Cuerpos y sexualidades en la escuela: De la "normalidad" a la disidencia (pp. 149-173). Paidós.

Burt, T., Gelnaw, A. y Lesser, L. K. (2010). Creating welcoming and inclusive environments for lesbian, gay, bisexual, and transgender (LGBT) families in early childhood settings. yC Young Children, 65(1), 97.

Butler, J. (1990). El género en disputa: el feminismo y la subversión de la identidad. Paidós.

Butler, J. (2001). Mecanismos psíquicos del poder: teorías sobre la sujeción (vol. 68). Universitat de València.

Campanella, M. (2018). Disrupción (en la educación): un enigma de tres pasos. http://pansophia.org/disrupcion-en-la-educacion-un-enigmade-tres-paso/

De Beauvoir, S. (1981). El segundo sexo. Siglo xx. Publicado originalmente en 1949.

Elizalde, S. (2014). Aprendiendo a ser mujeres y varones jóvenes: Prácticas de investimento del género y la sexualidad en la institución escolar. Intersecciones en Comunicación 8, 31-50.

Fabris, E. T. H., de Amorim Marcello, F. y Sommer, L. H. (2011). Crisis de la infancia moderna y nuevas configuraciones de la metáfora de la infancia. Educación y Pedagogía, 23(60), 89-99.

Frohard-Dourlent, H. (2016). 'I don't care what's under your clothes': The discursive positioning of educators working with trans and gender-nonconforming students. Sex Education, 16(1), 63-76.

Hernández, A. y Reybet, C. (2006). Acerca de masculinidades, feminidades y poder en las escuelas. Anales de la Educación Común, Tercer Siglo, 2(4), 128-135.

Ingrey, J. C. (2012). The public school washroom as analytic space for troubling gender: Investigating the spatiality of gender through students' self-knowledge. Gender and Education, 24(7), 799-817.

Kintner-Duffy, V. L., Vardell, R., Lower, J. K. y Cassidy, D. J. (2012). "The changers and the changed": Preparing early childhood teachers to work with lesbian, gay, bisexual, and transgender families. Journal of Early Childhood Teacher Education, 33(3), 208-223. 
Lamas, M. (2000). Diferencias de sexo, género y diferencia sexual. Cuicuilco, $7(18), 0$.

Lenzi, M. J. (2018). The trans athlete dilemma: A Constitutional analysis of high school transgender student-athlete policies. American University Law Review, 67, 841.

Ley 26.618 de matrimonio civil. (2010). Boletín Oficial de la República Argentina. http://servicios.infoleg.gob.ar/infolegInternet/anexos/165000169999/169608/norma.htm

Ley 26.743 de identidad de género. (2012). Boletín Oficial de la República Argentina. http://servicios.infoleg.gob.ar/infolegInternet/ anexos/195000-199999/197860/norma.htm

Mead, M. (2002). Cultura y compromiso. Gedisa.

Morgade, G. y Alonso, G. (2008). Educación, sexualidades, géneros: Tradiciones teóricas y experiencias disponibles en un campo en construcción. En G. Morgade y G. Alonso (comps.), Cuerpos y sexualidades en la escuela: De la "normalidad" a la disidencia (pp. 19-39). Paidós.

Muñoz Hurtado, L. V. (2019). El devenir de la autoridad pedagógica en Colombia: Emergencia, mitos y crisis [tesis de doctorado]. Universidad Pedagógica Nacional, Bogotá, Colombia.

Narodowski, M. (1994). Infancia y poder: La conformación de la pedagogía moderna. Aique.

Narodowski, M. (1999). Después de clase: Desencantos y desafíos de la escuela actual. Novedades Educativas.

Narodowski, M. (2016). Un mundo sin adultos. Debate.

Parga Romero, L. (2008). La construcción de los estereotipos del género femenino en la escuela secundaria. Universidad Pedagógica Nacional.

Payne, E. y Smith, M. (2014). The big freak out: Educator fear in response to the presence of transgender elementary school students. Journal of Homosexuality, 61(3), 399-418.

Postman, N. (1994). The disappearance of childhood. Vintage Books.

Rahilly, E. P. (2015). The gender binary meets the gender-variant child: Parents' negotiations with childhood gender variance. Gender \& Society, 29(3), 338-361.

Sabsay, L. (2009). Judith Butler para principiantes. Diario Página 12. https://www.pagina12.com.ar/diario/suplementos/soy/1-742-200905-08.html

Saldivia Menajovsky, L. (2016). El reconocimiento del derecho a la identidad de género de Luana. En V. Pavan (comp.), Niñez trans: Experiencia de reconocimiento y derecho a la identidad (pp. 77-87). Ediciones UNGS.

Scharagrodsky, P. (1999). Cuerpo, género y poder en la escuela: la construcción de la masculinidad en las clases de Educación Física [tesis de maestría]. Flacso. 
Scharagrodsky, P. A. y Narodowski, M. (2005). Investigación educativa y masculinidades: Más allá del feminismo, más acá de la testosterona. Revista Colombiana de Educación, 49, 61-80.

Sedgwick, E. K. (1993). 1993. Epistemology of the closet. En H. Abelove, M. Aina Barale, D. M. Halperin, The Lesbian and Gay Studies Reader (pp. 45-61). Routledge.

Slater, J., Jones, C. y Procter, L. (2018). School toilets: Queer, disabled bodies and gendered lessons of embodiment. Gender and Education, 30(8), 951-965.

Tabbush, C., Díaz, M. C., Trebisacce, C. y Keller, V. (2016). Matrimonio igualitario, identidad de género y disputas por el derecho al aborto en Argentina: La política sexual durante el kirchnerismo (2003-2015). Sexualidad, Salud y Sociedad, 22, 22-55.

Tomasini, M. (2008). Categorización sexual y socialización escolar en el nivel inicial. En G. Morgade y G. Alonso (comps.), Cuerpos y sexualidades en la escuela: De la "normalidad" a la disidencia (pp. 93-112). Paidós.

United Nations Children's Fund-Unicef. (1989). Convención sobre los Derechos del Niño. 